\title{
Comparison of ultraviolet Bi-directional Reflectance Distribution Function (BRDF) measurements of diffusers used in the calibration of the Total Ozone Mapping Spectrometer (TOMS)
}

\author{
James J. Butler ${ }^{* a}$, Hongwoo Park ${ }^{\mathrm{b}}$, P. Yvonne Barnes ${ }^{* *}$, Edward A. Early ${ }^{\mathrm{c}}$, Carina van Eijk- \\ Olij $^{\mathrm{d}}$, A. Erik Zoutman ${ }^{* * *}$, Stefanie van Buller-Leeuwen ${ }^{\mathrm{d}}$, Jos Groote Schaarsberg ${ }^{\mathrm{d}}$ \\ aaNASA's Goddard Space Flight Center, Code 920.1, Greenbelt, MD 20771; ${ }^{b}$ NASA's Goddard Space \\ Flight Center, Code 556, Greenbelt, MD 20771; ${ }^{\mathrm{c}}$ National Institute of Standards and Technology, \\ Optical Technology Division, Gaithersburg, MD 20899 USA; ${ }^{\mathrm{d}}$ TPD TNO, P.O. Box 155, 2600 AD, \\ Delft, The Netherlands;
}

\begin{abstract}
The measurement and long-term monitoring of global total ozone by ultraviolet albedo measuring satellite instruments require accurate and precise determination of the Bi-directional Reflectance Distribution Function (BRDF) of laboratorybased diffusers used in the pre-launch calibration of those instruments. To assess the ability of laboratories to provide accurate UltraViolet (UV) diffuse BRDF measurements, a BRDF measurement comparison was initiated by the NASA Total Ozone Mapping Spectrometer (TOMS) Project. From December 1998 to September 1999, NASA's Goddard Space Flight Center (GSFC), TPD TNO (formerly the TNO Institute of Applied Physics), and the National Institute of Standards and Technology (NIST) made BRDF measurements on four Spectralon diffusers used in the pre-launch calibration of three TOMS instruments. The diffusers were measured at the six TOMS wavelengths and at the incident and scatter angles used in the TOMS pre-launch calibration. The participation of GSFC, TPD TNO, and NIST in the comparison establishes a link between the diffuser calibrations of the on-orbit TOMS instruments, the Ozone Monitoring Instrument (OMI), and a national standards laboratory. The results of the comparison show that all of the BRDF measurements on the four diffusers agreed within $+0.85 \%$ to $-1.10 \%$ of the average BRDF and were well within the combined measurement uncertainties of the participating laboratories.
\end{abstract}

\section{INTRODUCTION}

The Total Ozone Mapping Spectrometer (TOMS), launched on October 24, 1978 aboard Nimbus 7, was a key instrument in the initial measurements of global atmospheric total column ozone. Except for a brief period in the mid1990's, the NIMBUS 7 TOMS, also known as Flight Model 1 (FM 1), and three follow-on TOMS, FMs 2 through 4 , have provided nearly 24 years of continuous global total ozone data, as summarized in Table 1 . The global total ozone data set will be extended by the Ozone Monitoring Instrument (OMI) aboard the Earth Observing System (EOS) Aura satellite beginning in 2004 and by the instruments of the National Polar Orbiting Environmental Satellite System (NPOESS) beginning in 2009. A long-term global ozone data set is essential in monitoring global ozone trends because of the concern that man-made chemicals are destroying the stratospheric ozone layer. Since the data set was and will continue to be built from the measurements of different instruments, the continuity of calibration and cross-calibration among these instruments is critical for correct scientific interpretation and sound policy formulation.

\footnotetext{
* butler@ltpmail.gsfc.nasa.gov; phone 301-614-5942; fax 301-614-5970; NASA’s Goddard Space Flight Center, Code 920.1, Greenbelt, MD 20771; ${ }^{* *}$ current address: Johns Hopkins University, School of Medicine, Brady Building, 600 N. Wolfe Street, Baltimore, MD 21287; ${ }^{* * *}$ current address: Science and Technology BV, P.O. Box 608, 2600 AP Delft, The Netherlands
} 
Table 1. Total Ozone Mapping Spectrometers: 1978 to Present

\begin{tabular}{|c|c|c|c|c|}
\hline Instrument & $\begin{array}{c}\text { Flight } \\
\text { Model } \\
\text { (FM) }\end{array}$ & Launch Date & End of Life & Data Record \\
\hline Nimbus 7 TOMS & 1 & $10 / 24 / 78$ & $5 / 6 / 93$ & $10 / 31 / 78$ to $5 / 6 / 93$ \\
\hline Meteor 3 TOMS & 2 & $8 / 15 / 91$ & $12 / 27 / 94$ & $8 / 22 / 91$ to11/24/94 \\
\hline $\begin{array}{c}\text { Earth Probes } \\
\text { TOMS }\end{array}$ & 3 & $7 / 2 / 96$ & Still operating & $7 / 25 / 96$ to present \\
\hline ADEOS TOMS & 4 & $8 / 17 / 96$ & $6 / 30 / 97$ & $9 / 11 / 96$ to $6 / 30 / 97$ \\
\hline QuikTOMS & 5 & $9 / 21 / 01$ & $9 / 21 / 01$ & Did not reach orbit \\
\hline
\end{tabular}

The primary input in TOMS ozone measurements is the ratio of the Earth's spectral radiance to the solar spectral irradiance. Solar radiation arriving at the Earth is absorbed, scattered, and reflected by the Earth's surface and its atmosphere. In the TOMS, the atmospheric column of ozone is deduced from the ratio of backscattered radiance to solar irradiance measured at six wavelengths between $308.6 \mathrm{~nm}$ and $360.0 \mathrm{~nm}$. Thus, the TOMS has radiance and irradiance measurement modes and must be calibrated for each. In principle, the uncertainties associated with measuring radiance and irradiance should cancel in the TOMS ratio measurement approach.

The pre-launch, radiance calibration of the TOMS instruments has used four laboratory Spectralon ${ }^{\mathrm{TM}}$ diffusers ${ }^{1}$ to convert spectral irradiance source standards to spectral radiance source standards ${ }^{2}$. The accuracy of the bi-directional reflectance distribution function (BRDF) of these diffusers limits the uncertainty of the calibration of the radiance measurement mode of the TOMS instruments and the accuracy of the ratio of the calibration of the radiance measurement mode to that of the irradiance measurement mode. In other words, the calibration uncertainty due to BRDF is not cancelled in the TOMS ratio calibration. Thus, the accuracy of the diffuser BRDF measurements directly affects the accuracy of the ratio measurement, the ozone retrieval, and the resulting ozone trends analyses ${ }^{3}$.

The BRDF of the diffusers used in the calibration of TOMS FM 3, 4, and 5 has been measured at NASA's GSFC's Diffuser Calibration Facility (DCaF) since 1993. These diffusers were measured at the National Institute of Standards and Technology (NIST) in 1999 to validate the GSFC measurements. The TOMS diffusers were also measured at the Netherland's TPD TNO in 1999 as an additional validation. These cross-calibrations have significance since the calibration and BRDF diffuser measurements for the OMI instrument will be performed at TPD TNO. These crosscalibrations effectively provide a direct comparison between the TOMS and OMI pre-launch calibrations. This paper presents the results of a comparison of the BRDF measurements of the four laboratory TOMS diffusers made at the three institutions from December 1998 through September 1999. The BRDF values were measured by each institution at the six TOMS wavelengths between $308 \mathrm{~nm}$ and $360 \mathrm{~nm}$ and at the incident and reflection angles of the TOMS pre-launch calibration geometry.

\section{EXPERIMENTAL PROCEDURE}

Reflectance properties of a sample are completely described by its BRDF. According to Nicodemus ${ }^{4}$, the BRDF, designated as $\mathrm{f}_{\mathrm{r}}$ in the equation below, is given by

$$
f_{\mathrm{r}}\left(\theta_{\mathrm{i}}, \varphi_{\mathrm{i}} ; \theta_{\mathrm{s}}, \varphi_{\mathrm{s}} ; \lambda\right)=\mathrm{d} L_{\mathrm{r}}\left(\theta_{\mathrm{i}}, \varphi_{\mathrm{i}} ; \theta_{\mathrm{s}}, \varphi_{\mathrm{s}} ; \lambda\right) / \mathrm{d} E_{\mathrm{i}}\left(\theta_{\mathrm{i}}, \varphi_{\mathrm{i}} ; \lambda\right)
$$

where $\mathrm{d} L_{\mathrm{r}}$ is the reflected radiance, $\mathrm{d} E_{\mathrm{i}}$ is the incident irradiance, $\theta$ is the polar angle, $\varphi$ is the azimuthal angle, the subscripts $i$ and $\mathrm{s}$ refer to the incident and scatter directions, and $\lambda$ is the wavelength. For the comparison presented in this paper, all participating laboratories made absolute measurements of $f_{\mathrm{r}}$ in which both $\mathrm{d} L_{\mathrm{r}}$ and $\mathrm{d} E_{\mathrm{i}}$ were directly measured $^{5}$.

Four Spectralon samples were used in the comparison. Three of the samples (i.e. TOMS Spectralon diffuser numbers $\mathrm{S} / \mathrm{N}$ 001, S/N 002, and S/N 003) were used in the pre-launch radiance calibration of the TOMS FM 3, FM 4, and FM 5 
instruments. These diffusers are $8.9 \mathrm{~cm}$ in diameter, mounted in a $10.7 \mathrm{~cm}$ by $10.7 \mathrm{~cm}$ black-anodized aluminum holder, and secured using two set-screws located at adjacent corners of the holder. The diffusers were illuminated from the direction opposite the set-screws, and the in-plane, forward scattered light was measured. The fourth sample was a 30.5 $\mathrm{cm}$ by $30.5 \mathrm{~cm}$ Spectralon diffuser, designated H4, obtained from the GSFC Radiometric Calibration and Development Facility (RCDF). This diffuser was used in the calibration of TOMS FM 5, the Solar Backscatter UltraViolet/2 (SBUV/2) instrument, the Shuttle Solar Backscatter Ultraviolet (SSBUV) instrument, the Shuttle Ozone Limb Sounding Experiment (SOLSE) and the Limb Ozone Retrieval Experiment (LORE). H4 has three, small, triangularly-oriented holes located at the center of one side and at two adjacent corners of the square diffuser. This diffuser was normally illuminated. With the diffuser oriented such that the side-centered hole is at the top and two corner holes are at the bottom, the scattered light was measured from the diffuser's right side.

The wavelengths, bandwidth, incident, and scatter angles of the diffusers in the comparison were identical to those used in the calibration of the TOMS satellite instruments; and all BRDF values are reported for the unpolarized scattering case. These parameters summarized in Table 2 .

Table 2. Experimental Parameters for the BRDF Comparison on TOMS Spectralon Diffusers S/N001, 002, 003, and $\mathrm{H} 4$

\begin{tabular}{|l|c|}
\hline \multicolumn{1}{|c|}{ Parameter } & Value \\
\hline Wavelengths (nm) & $308.6,312.5,317.5,322.3,331.2,360$ \\
\hline Bandwidth (nm) & 10 \\
\hline Incident polar angle (deg) & 41 \\
S/N 001, 002, \& 003 & 0 \\
H4 & 180 \\
\hline Incident azimuthal angle (deg) & 49 \\
\hline Scatter polar angle (deg) & 33 \\
S/N 001, 002, \& 003 & 0 \\
\hline H4 & Unpolarized scattering case \\
\hline Polarization azimuthal angle (deg) & \\
\hline
\end{tabular}

The following instruments were employed in the BRDF comparison: the NIST Spectral Tri-Function Automated Reference Reflectometer (STARR), the scatterometer located in the TPD TNO Absolute Radiometric Calibration Facility (ARCF), and the scatterometer located in NASA's GSFC Diffuser Calibration Facility (DCaF). These instruments and associated measurement techniques are briefly described below.

\subsection{NIST'S STARR REFLECTOMETER}

The NIST STARR instrument [6] is located in a black-walled laboratory with a ventilation system equipped with special air filters. The illuminator consists of a feedback-stabilized $150 \mathrm{~W}$ xenon arc lamp, a $1 / 4 \mathrm{~m}$ Czerny-Turner monochromator, focusing and fold mirrors, and a Glan-Taylor polarizer. This illuminator produces polarized radiant flux with a spectral bandwidth of $10 \mathrm{~nm}$ and a $17 \mathrm{~mm}$ diameter illuminated area on the samples. The receiver consists of a precision circular aperture of diameter $31.85 \mathrm{~mm}$, a fused silica lens, and a $10 \mathrm{~mm}$ by $10 \mathrm{~mm}$ ultraviolet-enhanced silicon photodiode. Measurements were made for both s- and p-polarizations of the incident flux, and the scatter for unpolarized radiant flux was calculated from the average for these two cases.

The STARR instrument is capable of making scatter measurements in both absolute mode and relative to a sample with known BRDF. The absolute scatter measurement mode was used exclusively in this study. In this mode, the incident and reflected radiant fluxes are measured, and the BRDF is calculated from the ratio of these fluxes divided by the projected solid angle from the sample to the receiver aperture. The optical prescriptions for the illuminator and receiver lead to scatter measurements in which the field-of-view of the receiver is under-filled by the illuminated area on the sample. The expanded uncertainty [7] $(k=2)$ for the BRDF measurements made in this study is $0.004 \mathrm{sr}^{-1}$. 


\subsection{TPD TNO ARCF SCATTEROMETER}

The TNO Institute of Applied Physics scatterometer ${ }^{8}$ is housed in a black-walled, class 100 cleanroom. In the TNO scatterometer, the output of a $300 \mathrm{~W}$ xenon arc lamp is collimated and focused onto the entrance slit of a double prism monochromator. The bandwidth of the exiting beam is $20 \mathrm{~nm}$. The exiting beam is collimated using a second spherical mirror producing a beam with a maximum diameter of $40 \mathrm{~mm}$. The collimated beam power is monitored using a quartz window to reflect a fraction of the beam into a reference detector. The remaining transmitted portion of the beam, with $\mathrm{s}$ - and p- polarization equal to better than $0.5 \%$, is sent either to the sample or to the scatter detector. The ARCF goniometer is mounted on a self-leveling, vibrationally isolated optical table. The goniometer sample stage is capable of three-axis rotation and one-dimensional translation, providing a complete out-of-plane measurement capability. The receiver is mounted on an arm capable of rotation in a horizontal plane over 360 degrees. In this study, the receiver consisted of an entrance aperture, an imaging mirror, a variable field of view aperture, and a silicon photodiode detector positioned 12 degrees off-axis. Light reflected from the silicon photodiode is collected and effectively absorbed using three Schott NG1 filters in a trap configuration. The field of view of the scatter detector is determined to an uncertainty of better than $0.01 \%$.

The ARCF scatterometer is capable of making scatter measurements in absolute mode and relative to a sample with known BRDF. The absolute scatter measurement mode was used in this study. The optical prescriptions for the source and receiver lead to scatter measurements in which the field-of-view of the ARCF receiver is over-filled by the illuminated area on the sample under test. The measurement uncertainty for the ARCF BRDF measurements is 0.0034 $\mathrm{sr}^{-1}(k=2)$.

\subsection{NASA's GSFC SCATTEROMETER}

The NASA's GSFC scatterometer ${ }^{9}$ is located in a black-walled, class 10,000 cleanroom. The DCaF scatterometer light source subsystem is mounted on a vertically-oriented cleanroom-compatible optical table and is comprised of a $75 \mathrm{~W}$ xenon arc lamp and a $1 / 4 \mathrm{~m}$ Czerny-Turner monochromator. A He/Ne laser at $632.8 \mathrm{~nm}$ is used for sample alignment. A 600 lines $/ \mathrm{mm}$ monochromator grating blazed at $200 \mathrm{~nm}$ produces an exiting light bandwidth of $10 \mathrm{~nm}$. The output beam from the monochromator is focussed by a spherical mirror onto a $3 \mathrm{~mm}$ diameter circular aperture and chopped. The chopped beam is monitored using a silicon photodiode reference detector. Following the circular aperture, the beam is linearly polarized using a Glan-Taylor polarizer and focused on the detector by a second spherical mirror. A pair of folding mirrors directs the beam onto the horizontally oriented sample stage while maintaining its linear polarization. The scatterometer optical system produces a $14 \mathrm{~mm}$ by $8 \mathrm{~mm}$ normally illuminated area on the sample. Unpolarized BRDF measurements are obtained by using a polarization insensitive detector and by averaging the two linear, crossed polarization scattering measurements. The goniometer consists of a horizontally-oriented sample stage and a detector rotation-translation stage. The detector rides on a rotatable half circle arc mechanism. The detector can move 180 degrees along the arc relative to the sample normal and to the origin of the sample stage. This combination of sample stage and detector goniometer provides a complete out-of-plane scatter measurement capability. For the measurements presented here, an ultraviolet sensitive silicon photodiode detector was used. This receiver subsystem also includes an aperture stop, field lens, field stop, and a pre-amplifier.

The DCaF scatterometer is capable of making absolute and relative scatter measurements with the absolute scatter measurement mode used in this study. For consistency, the scatterometer measurements were compared before and after the comparison to measurements made in the NIST STARR facility on two Spectralon samples. Similar to the NIST STARR instrument, the optical prescriptions for the source and receiver lead to scatter measurements in which the field of view of the GSFC scatterometer receiver is underfilled by the illuminated area on the sample under test. The uncertainty of the BRDF measurements from the NASA scatterometer is $0.0047 \mathrm{sr}^{-1}(k=2)$. 


\section{RESULTS}

For this comparison, the BRDF of the four diffusers was measured by GSFC in December 1998, by TPD TNO in February 1999, by NIST in March 1999, and again by GSFC in September 1999. The results of the BRDF measurements on the TOMS Spectralon Diffusers S/N 001, 002, and 003 and on the RDCF Diffuser H4 are shown in Figures 1a through d. The data in Figures 1a, b, and c were acquired at the 6 TOMS wavelengths of $308.6 \mathrm{~nm}, 312.5$ $\mathrm{nm}, 317.5 \mathrm{~nm}, 322.3 \mathrm{~nm}, 332.1 \mathrm{~nm}$, and $360.0 \mathrm{~nm}$ and at the TOMS calibration geometry of 41 degrees incidence and 49 degrees scatter, as measured relative to the diffuser normal. The data of Figure 1d were acquired at the same 6 TOMS wavelengths and at normal illumination and 33 degrees scatter. Figures 2, 3, and 4 show the percent difference from the average measured BRDF of the GSFC, TPD TNO, and NIST measurements.
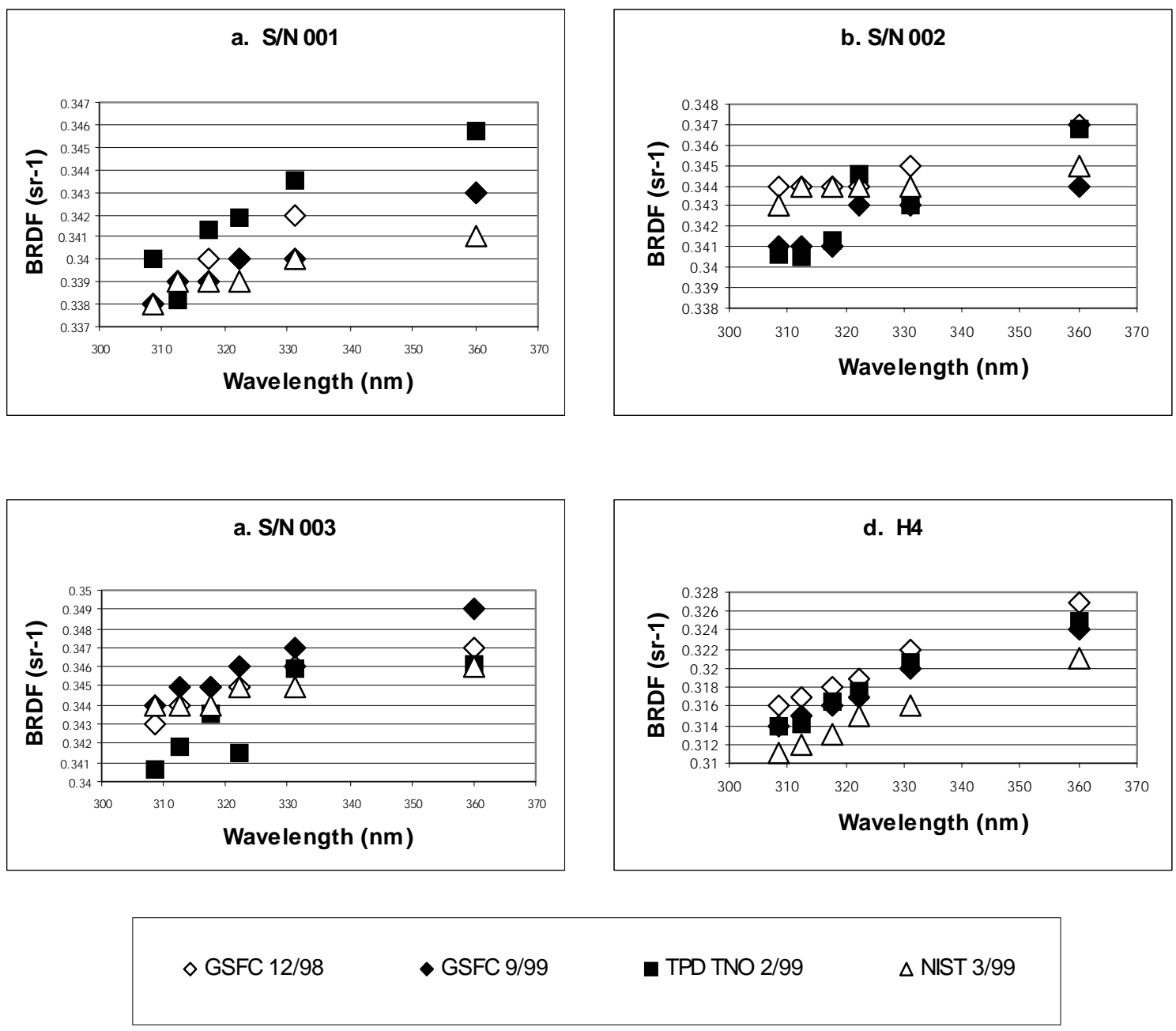

Figure 1. BRDF measured by GSFC, TPD TNO, and NIST on TOMS Spectralon diffusers S/N001, S/N002, S/N003 and on RDCF Spectralon diffuser H4. 
NASA's GSFC
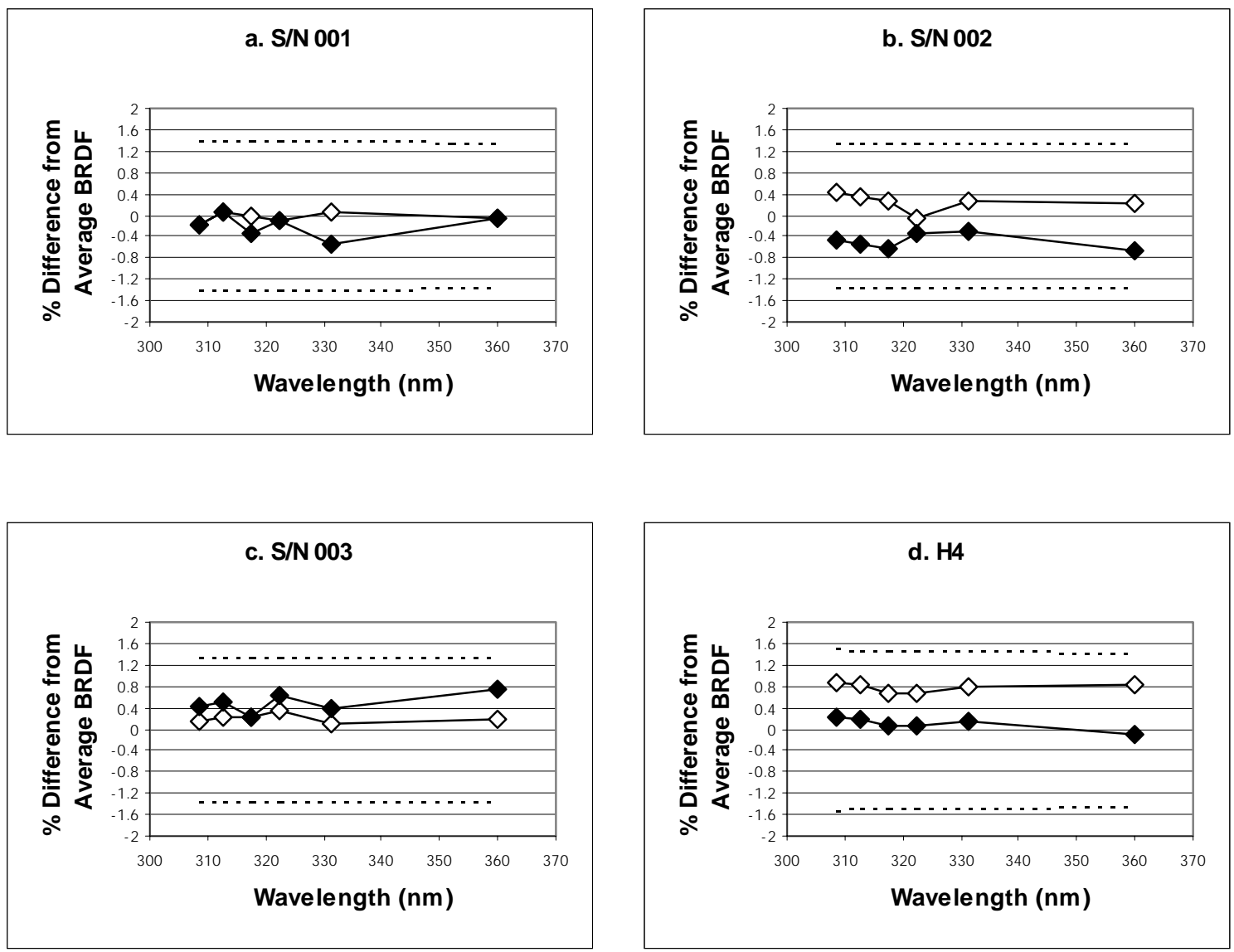

Figure 2. Percent difference of the GSFC BRDF measurements from the average BRDF measured by the comparison participants. The white diamonds are data acquired in December 1998; the black diamonds are data acquired in September 1999. The dashed lines represent the $k=2$ expanded uncertainty of the GSFC measurements. 


\section{TPD TNO}
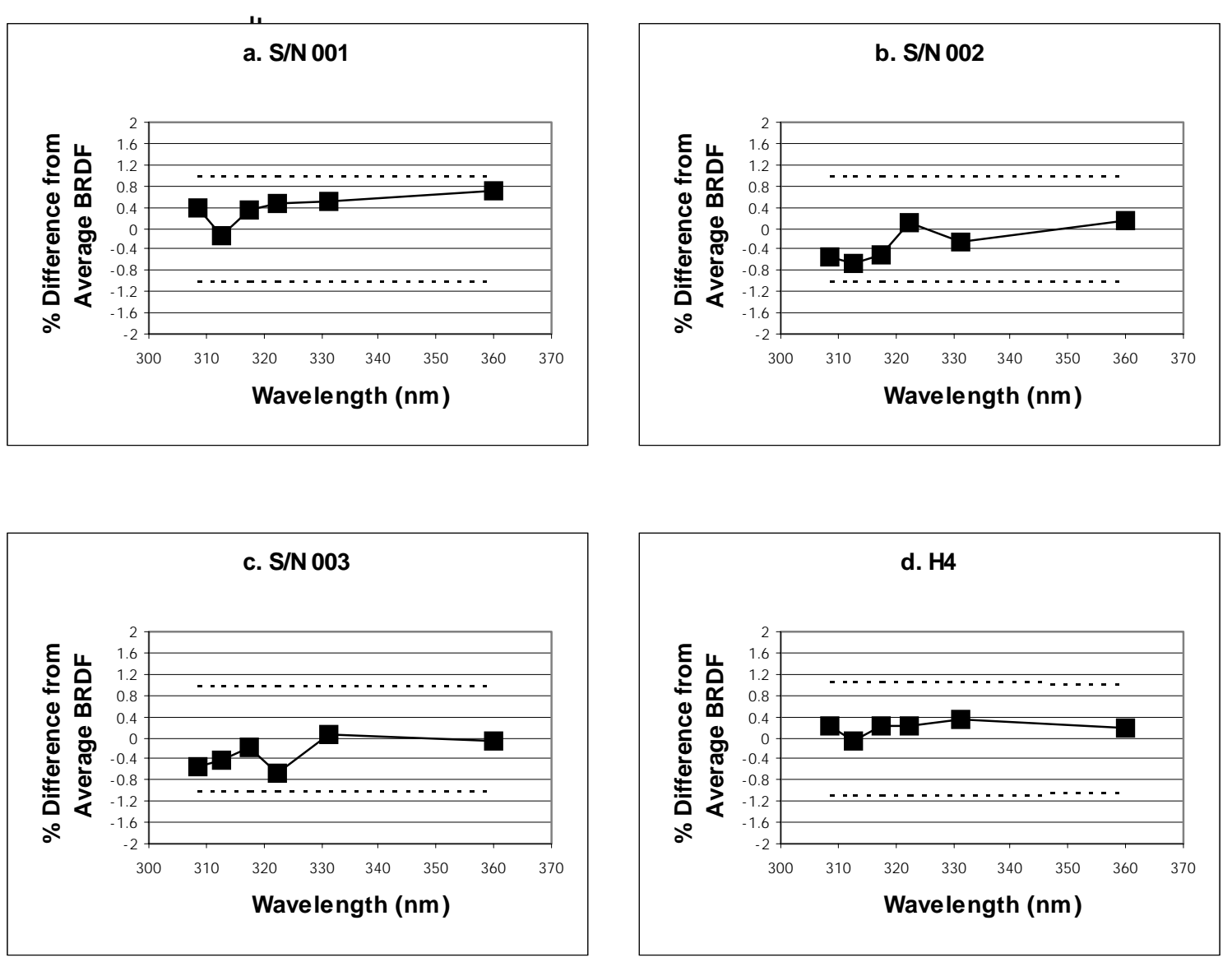

Figure 3. Percent difference of the TPD TNO BRDF measurements from the average BRDF measured by the comparison participants. The dashed lines represent the TPD TNO expanded measurement uncertainty $(k=2)$. 

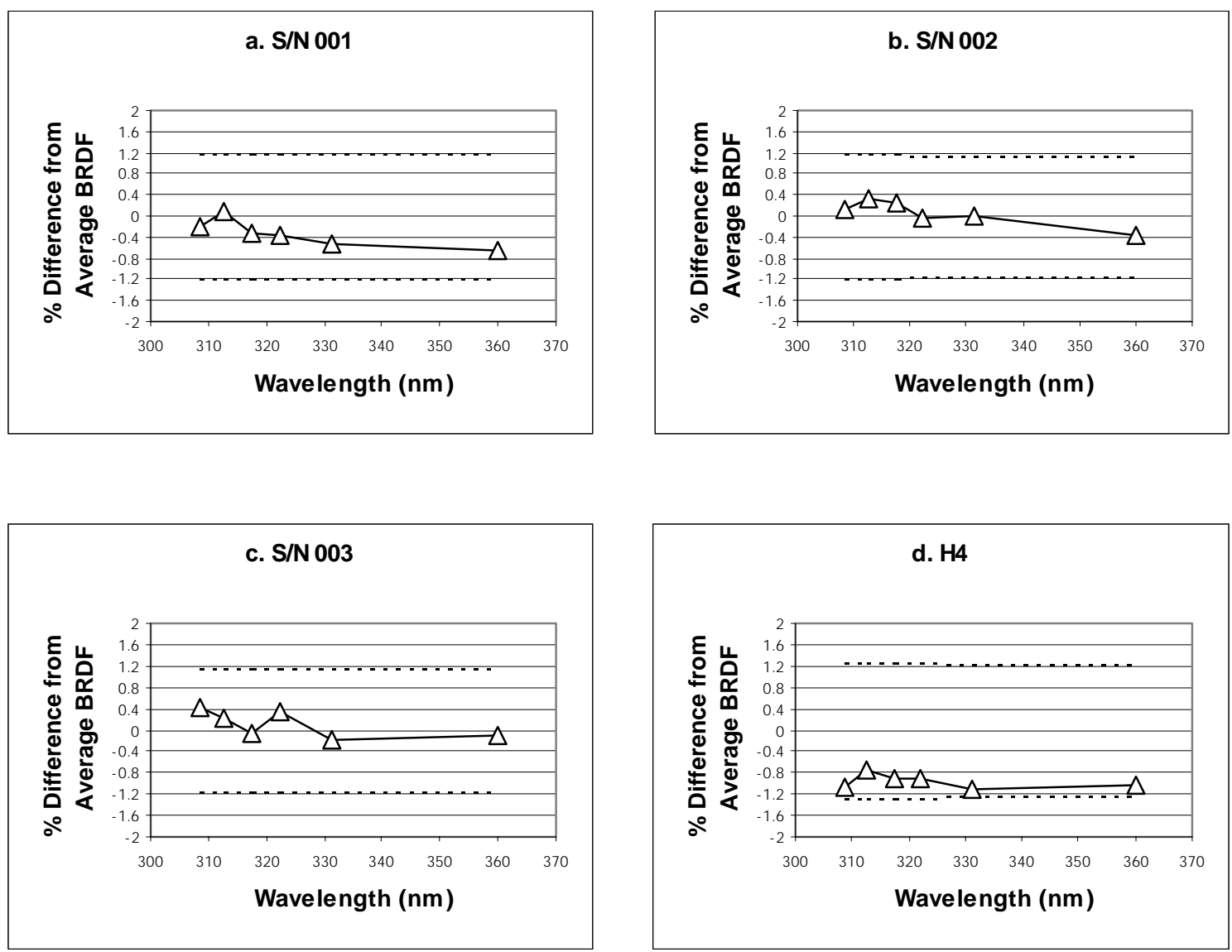

Figure 4. Percent difference of the NIST BRDF measurements from the average BRDF measured by the comparison participants. The dashed lines represent the NIST expanded measurement uncertainty $(k=2)$.

\section{DISCUSSION}

All of the BRDF measurements shown in Figures 1a through d show excellent agreement, well within the combined measurement uncertainties of the participating laboratories. The largest difference between individual laboratories is seen in the GSFC December 1998 and the NIST March 1999 data. These data show an average difference of 1.73\%. The NIST and GSFC BRDF data shown in Figures 1a through d show a slowly increasing trend with increasing wavelength. While the majority of the TPD TNO BRDF data exhibits a similar behavior with wavelength, several points, such as those at $312.5 \mathrm{~nm}$ for $\mathrm{S} / \mathrm{N} 001$, at $331.2 \mathrm{~nm}$ for $\mathrm{S} / \mathrm{N} \mathrm{002}$, and at $322.3 \mathrm{~nm}$ for S/N 003, do not follow that trend. The source of these deviations currently is the subject of additional study by TPD TNO.

The excellent agreement between the BRDF measurements is more clearly illustrated in Figures 2 through 4, in which the percent difference of each participant's measurements from the average of all the BRDF measurements is shown for all wavelengths. The agreement between all of the participants' measurements and the average is within $+0.85 \%$ to $1.10 \%$. A fraction of the disagreement between the individual laboratory measurements can be attributed to the laboratories illuminating and viewing different areas of the diffusers with slightly different reflectance. Unfortunately, the BRDF of the four diffusers was not spatially mapped in this study to quantify that effect. 
GSFC was the only laboratory to measure the diffusers twice, once in December 1998 and again in September 1999. As illustrated in Figures 2a through d, these two sets of measurements agree to within the combined $(k=2)$ measurement uncertainty of the GSFC scatterometer; and the differences appear to be due to a combination of random and systematic errors. The biggest differences between the GSFC December 1998 and September 1999 data are seen in Figures 2b and $2 \mathrm{~d}$ and range from $0.3 \%$ to $1.0 \%$.

For the S/N 001, 002, and 003 diffusers, the agreement between the average of all GSFC measurements at all wavelengths and the average of all the participants' measurements is $0.13 \%$ for the December 1998 data and $-0.06 \%$ for the September 1999 data. For diffuser H4, the agreement between the GSFC measurements averaged at all wavelengths and the average measurements of all the participants is $0.77 \%$ for the December 1998 data and $0.09 \%$ for the September 1999 data. Differences between the two GSFC measurements and between the GSFC measurements and those of TPD TNO and NIST on the S/N 001, 002, and 003 diffusers are primarily due to non-reproducibility in sample alignment and to errors in the measurement of incident power. These diffusers were measured in a near-specular portion of the Spectralon BRDF curve in which the BRDF is rapidly rising with increasing scatter angle. At this measurement geometry, small errors in sample alignment can easily lead to appreciable differences in measured BRDF. Since H4 was illuminated normal to the diffuser, differences in the GSFC measurements on $\mathrm{H} 4$ are believed to be predominantly due to errors in the measurement of incident power.

The TPD-TNO measurements on S/N 001, S/N 002, and S/N 003 show good agreement with the average measured BRDF. For those diffusers, the agreement between the TPD TNO measurements averaged over all wavelengths and the global average is $-0.07 \%$. For H4, the agreement averaged over all the TPD TNO measurements at all wavelengths with the global average is $0.15 \%$.

The NIST BRDF measurements on S/N 001, S/N 002, and S/N 003 also show good agreement with the average measured BRDF. For those diffusers, the agreement between the NIST measurements averaged over all wavelengths and the global average is $-0.06 \%$. For H4, the agreement between the NIST measurements averaged over all wavelengths and the global average is $-0.95 \%$, ranging between $-0.8 \%$ to $-1.1 \%$. The fact that the GSFC, TPD TNO, and NIST measurements on $\mathrm{H} 4$ all show the same spectral shape with wavelength suggests a systematic source for the differences between these measurements.

\section{CONCLUSION}

The comparison of BRDF measurements by NASA's GSFC, TPD TNO, and NIST on four Spectralon diffusers used in the pre-launch calibration of three TOMS satellite instruments agreed to within $0.85 \%$ to $-1.10 \%$ of the average BRDF computed from all the participants' measurements. All BRDF measurements were well within the combined measurement uncertainties $(k=2)$ of the participating institutions. This comparison establishes the level of agreement between the BRDF measurements of NASA's TOMS Project, the EOS OMI Project, and the national standards laboratory of the United States.

\section{REFERENCES}

1. Certain commercial equipment, instruments, or materials are identified in this paper to foster understanding. Such identification does not imply recommendation or endorsement by the National Institute of Standards and Technology or by the National Aeronautics and Space Administration, nor does it imply that the materials or equipment identified are necessarily the best available for the purpose.

2. H. Park, A. Krueger, E. Hilsenrath, G. Jaross, and R. Haring, "Radiometric Calibration of Second Generation Total Ozone Mapping Spectrometer (TOMS)," Proc. SPIE, 2820, 162-173 (1996).

3. G. Jaross, A.J. Krueger, and C. Wellemeyer, "Sensitivity of Total Ozone Mapping Spectrometer Products to Diffuse Reflectance Measurements," Metrologia, 35, 663-668 (1998). 
4. F.E. Nicodemus, J.C. Richmond, J.J Hsia, I.W. Ginsberg, and T. Limperis, "Geometrical Considerations and Nomenclature for Reflectance," NBS Monograph, No. 160, U.S. Department of Commerce, National Bureau of Standards, $52 \mathrm{pp}$.

5. "ASTM E1392-90, Standard Practice for Angle Resolved Optical Scatter Measurements on Specular or Diffuse Surfaces," ASTM Standards on Color and Appearance Measurments, $5^{\text {th }}$ ed., American Society for Testing and Materials, 439-448 (1997).

6. B.N. Taylor and C.E. Kuyatt, "Guidelines for Evaluating and Expressing the Uncertainty of NIST Measurement Results," NIST Technical Note 1297, U.S. Department of Commerce, National Institute of Standards and Technology, 20 pp.

7. J.E. Proctor and P.Y. Barnes, "NIST High Accuracy Reference Reflectometer-spectrophotometer," J. Res. Natl. Inst. Stand. Technol., 101, 619-627 (1996).

8. C. Smorenburg, A.L.G. van Valkenburg, and H.G.C. Werij, “Absolute Radiometric Calibration Facility,” Proc. SPIE, 2583, 166-177 (1995).

9. T.F. Schiff, M.W. Knighton, D.J. Wilson, F.M. Cady, J.C. Stover, and J.J. Butler, "Design Review of a High Accuracy UV to Near IR Scatterometer,” Proc. SPIE, 1995, 121-130 (1993). 